\title{
L'acide $\gamma$-hydroxybutyrique (GHB) : un vieux produit, de nouveaux problèmes
}

\section{$\gamma$-hydroxybutyric acid $(G H B)$ : old substance, new problems}

Marc DEVEAUX*

Institut de Médecine Légale, Faculté de Médecine - F-59045 LILLE Cedex

* Auteur à qui adresser la correspondance : Marc DEVEAUX, Institut de Médecine Légale,

Faculté de Médecine, place de Verdun -F-59045 LILLE Cedex

Tél : 0320623510 - Fax : 0320623512 - e-mail : mdeveaux@easynet.fr

(Reçu le 16 octobre 2002 ; accepté le 29 octobre 2002)

\section{RÉSUMÉ}

Depuis une dizaine d'années, l'acide $\gamma$ hydroxybutyrique $(G H B)$ est présenté comme la nouvelle drogue à la mode dans les rave parties et dans le milieu culturiste. Avec ses dérivés la $\gamma$-butyrolactone (GBL) et le 1,4-butanediol (BD), ils sont utilisés également pour la soumission chimique. Ces produits datent des années 1960 où ils ne posaient aucun souci, leur marge thérapeutique étant assez grande, mais leur vogue actuelle dans les milieux festifs fait surgir le problème des intoxications et de leur diagnostic. Une implication de l'expert en toxicologie peut se voir également dans les cas de soumission chimique et de recherche de causes de la mort. Nous passons en revue les effets attendus, les effets secondaires et toxiques du GHB, de la GBL et du BD. Cette revue de la littérature fait également le point sur les méthodes analytiques et surtout sur l'interprétation des résultats. Les méthodes d'analyses par chromatographie gazeuse/spectrométrie de masse sont pratiquement standardisées. Selon les laboratoires, la limite de détection varie de 0,5 à $2 \mu \mathrm{g} / \mathrm{mL}$ et la limite de quantification de 2,5 à $5 \mu \mathrm{g} / \mathrm{mL}$.

\section{SUMMARY}

Since a dozen of years $\gamma$-hydroxybutyric acid $(G H B)$ is presented as very popular in rave-parties and for bodybuilders. Together with its derivatives $\gamma$-butyrolactone $(G B L)$ and 1,4butanediol $(B D)$, they are also used as date-rape drugs and by bodybuilders. They are old substance products, designed in the 60's, with a relatively favorable therapeutic beneficial effects, but new problems are appearing in rave-parties, with numerous intoxications, diagnosis of which is quite difficult. The toxicologist plays also an important role in case of facilitated sexual assault and determination of cause and manner of death. In this paper, we review therapeutic, side and toxic effects of $G H B, G B L$ and $B D$. This review leads also to know what is actually done in laboratories for determination in biological samples and toward the interpretation of the results. GC-MS analysis is quite the same in almost the labs. Detection limits range from 0.5 to $2 \mu \mathrm{g} / \mathrm{mL}$, quantification from 2.5 to $5 \mu \mathrm{g} / \mathrm{mL}$. Samples are very small, less than $50 \mu \mathrm{L}$. Interpreting the results is difficult, because $G H B$ is an endogenous substance, naturally found in blood and urine, with a 
Les prises d'essai sont faibles : 20 à $50 \mu L$ de sérum, plasma ou urine suffisent. L'interprétation des résultats est délicate car le GHB est une substance endogène que l'on retrouve dans le sang et les urines et son élimination est très rapide. On considère que les taux physiologiques restent inférieurs à $5 \mu \mathrm{g} / \mathrm{mL}$ dans le sang et $10 \mu \mathrm{g} / \mathrm{mL}$ dans l'urine. Chez un sujet décédé, tout résultat dans le sang doit être confirmé par un dosage dans l'urine et le vitré. La recherche du GHB dans les cheveux peut être également un atout précieux pour l'interprétation.

\section{MOTS-CLÉS}

$G H B, G B L, 1,4-B D$, soumission chimique,

\section{Introduction}

L'acide $\gamma$-hydroxybutyrique (GHB) est un acide gras à chaîne courte présent dans le système nerveux central et les tissus périphériques des mammifères. C'est un dérivé de l'acide $\gamma$-aminobutyrique (GABA). Synthétisé par Laborit (1), il a obtenu une autorisation de mise sur le marché en 1961, renouvelée en 1998 pour la sédation en neurotraumatologie et comme adjuvant anesthésique en chirurgie et obstétrique. En France, la spécialité Gamma-OH® injectable est un anesthésique inscrit sur la liste I des substances vénéneuses, dont l'usage est réservé au milieu hospitalier. Il existe une spécialité sous forme buvable commercialisée en Italie (Alcover(B), disposant d'une autorisation temporaire d'utilisation nominative en France pour le traitement de la narcolepsie avec catalepsie ; elle est inscrite sur la liste des stupéfiants et son obtention est assez complexe (2). Un produit analogue, le Xyrem®, est soumis à la réglementation des médicaments orphelins aux Etats-Unis (3). Le GHB a aussi été utilisé dans le sevrage de l'alcoolisme et des opiacés, et comme adjuvant dans le traitement de la schizophrénie (4). A la fin des années 1980, le GHB a été commercialisé comme complément alimentaire aux Etats-Unis : il était consommé par les culturistes pour stimuler l'hormone de croissance dans le but d'accroître la masse musculaire, et également pour augmenter le métabolisme des graisses (5). Malheureusement, cette idée n'a jamais été confirmée (4). La fréquence de son utilisation s'est accrue pendant les années 1990, car il avait acquis la réputation d'accroître la libido et de rendre les femmes plus vulnérables, surtout en association à de l'alcool.

L'ampleur du phénomène a conduit la Food and Drug Administration américaine (FDA) a interdire la vente du GHB en dehors de prescriptions médicales $(3,4)$.

Néanmoins sa vente continue illégalement, essentiellement par l'Internet $(6,7)$. L'observation attentive par les usagers de certains de ses effets secondaires (euphorie, relaxation, ivresse, somnolence, inconscience, short half-life. One must consider that physiological concentrations are less than $5 \mu \mathrm{g} / \mathrm{mL}$ in blood and less than 10 $\mu \mathrm{g} / \mathrm{mL}$ in urine. After death, a positive result in blood must be confirmed in determining $G H B$ in urine and vitreous. Determination of $G H B$ in hair can also be an asset for interpretation of the analytical data.

\section{KEY-WORDS}

$G H B, G B L, 1,4-B D$, date-rape drug.

amnésie), a fait que depuis quelques années, le GHB est utilisé en Europe comme euphorisant dans les rave parties et comme substance induisant une soumission chimique pour faciliter les viols. Les journalistes se sont fait l'écho de ces rumeurs, le plus souvent en les amplifiant (2). Certaines publications $(8,9)$ mettent en cause le GHB sans apporter la preuve formelle de sa présence dans le sang ou l'urine : il y a donc un débat largement ouvert sur la responsabilité du GHB dans ces affaires médicolégales, car il faut pouvoir mettre en évidence sa présence dans les prélèvements biologiques, le doser puis interpréter correctement les résultats. De même en cas de mort suspecte, la mise en évidence de GHB dans les prélèvements pose de gros problèmes d'interprétation à l'expert toxicologue. Les pièges apparaissent nombreux et nous les passerons en revue.

\section{Action du GHB}

\section{Rôle physiologique du GHB}

Il est bien établi que le GHB présente toutes les caractéristiques d'un neurotransmetteur, et on le retrouve en plus fortes concentrations dans l'hippocampe, l'hypothalamus, les ganglions basaux et la substance noire (revue générale dans (3)). Sa biosynthèse s'effectue à partir de l'acide glutamique, transformé en GABA puis en semi-aldéhyde succinique (SAS) par une GABA aminotransférase. Le SAS est transformé essentiellement en acide succinique puis incorporé dans le cycle de Krebs. Seule une faible partie ( $2 \%$ environ) est transformée en GHB par une SAS réductase spécifique. Il existe d'autres voies endogènes de production. Un alcool aliphatique, le 1,4-butanediol, se transforme en 4-hydroxybutanal puis en GHB sous l'action de l'ADH. Enfin, une lactone, la $\gamma$-butyrolactone, peut être transformée en GHB par une lactonase (10). La question de la réversibilité de cette réaction n'est pas encore tranchée.

Le GHB est libéré après la dépolarisation neuronale 
puis se lie de façon réversible à des récepteurs spécifiques. Il y a ensuite recapture dans la fente synaptique et dans le cytosol, une GHB déshydrogénasse catalyse sa transformation en SAS, puis en succinate. Il se transforme également en GABA par transamination, en glycolate puis acétate par béta-oxydation. Au total, $95 \%$ sont éliminés sous forme de $\mathrm{CO}_{2}$. On a montré que ce mécanisme était saturable et que la demi-vie d'élimination était dose dépendante (11). On peut donc raisonnablement s'attendre à ce qu'elle soit plus longue en cas de surdosage.

Les principaux effets neurologiques du GHB sont des modulations des neuromédiateurs classiques (3). Ses activités gabaergique, sérotoninergique, cholinergique et dopaminergique aboutissent à une diminution de la neurotransmission dopaminergique. D'autres études (12) montrent que dans une seconde phase, la libération de la dopamine est stimulée par le GHB dans le striatum et les structures corticolimbiques : ceci pourrait expliquer les effets euphorisants et addictogènes. Le doute subsiste encore à ce sujet.

Le GHB jouerait donc un rôle dans les cycles du sommeil, la régulation de la température corporelle, le métabolisme cérébral du glucose, la mémoire et la protection du SNC contre l'hypoxie $(4,13)$.

\section{Effets cliniques du GHB}

Nous ne nous intéresserons qu'à l'usage par voie orale du sel de sodium de l'acide $\gamma$-hydroxybutyrique, disponible sur le marché illicite sous forme de poudre ou de liquide. Seules les études contrôlées sont citées. Après ingestion, le GHB est rapidement absorbé et les premiers effets se font sentir après 15 à $30 \mathrm{~min}$. Le pic sanguin est atteint entre 20 et $45 \min (4,11,14-16)$. La demi-vie est très courte, dose dépendante : 20 à $40 \mathrm{~min}$ pour des posologies de 12,5 à $60 \mathrm{mg} / \mathrm{kg}$. L'élimination urinaire est faible (moins de $1 \%$ de la dose ingérée) et rapide, puisque le pic urinaire est atteint en moins de 4 h (17-19). On n'en détecte plus dans les urines au bout de $12 \mathrm{~h}$ pour des doses supérieures à $5 \mathrm{~g}(20)$. Des doses de 1 à $2 \mathrm{~g}$ sont indétectables après $10 \mathrm{~h}(21)$.

L'intensité des effets sur le SNC est fonction de la dose ingérée et elle est amplifiée par l'alcool et autres dépresseurs du SNC consommés simultanément (agonistes des opiacés, benzodiazépines, neuroleptiques). Les relations entre les doses, les effets et les concentrations sanguines sont données dans le tableau I. La marge séparant les effets euphorisant des effets indésirables est étroite mais la dose mortelle a été estimée entre 5 à 15 fois la dose procurant une perte de conscience. Des crises tonico-cloniques sont inconstamment observées en cas de surdosage $(3,22-24)$ et on a décrit des utilisateurs extrêmement agités et comme cherchant l'air désespérément (4). Les effets cardiovasculaires sont une hypotension et une bradycardie. Une hypersialorhée et des vomissements incoercibles ont été également décrits (7, 17, Goullé J.P. : communication personnelle). L'hypothermie $\left(<35^{\circ} \mathrm{C}\right)$ n'est pas rare. Il n'existe pas d'antidote connue actuellement.

Les autres effets recherchés lors d'un mésusage ou d'un abus sont surtout l'accroissement de la masse musculaire (5), mais cet effet est très controversé. De même, les effets positifs sur la libido semblent être plutôt subjectifs et liés à la desinhibition. Enfin le sentiment de bien-être et l'euphorie sont souvent décrits (25-27).

Tableau I : Relations entre la dose de GHB ingérée, les effets observés et la concentration sérique (d'après 2, 4, 7, $15,17,19,23,24)$.

\begin{tabular}{|c|c|c|}
\hline Dose ingérée (g) & Effet & $\begin{array}{c}\text { Concentration sérique } \\
(\mu \mathrm{g} / \mathrm{mL})\end{array}$ \\
\hline 0,7 à 1 & $\begin{array}{c}\text { amnésie antérograde, } \\
\text { hypotonie }\end{array}$ & \\
\hline 1,5 à 2,2 & $\begin{array}{l}\text { difficultés d'idéation, } \\
\text { vertiges, endormissement }\end{array}$ & $24-90$ \\
\hline 2,0 a 3,0 & $\begin{array}{l}\text { euphorie, ivresse, } \\
\text { perte de conscience }\end{array}$ & 50 \\
\hline$>3,5$ & anesthésie & $100-125$ \\
\hline$>4,2$ & $\begin{array}{l}\text { respiration de Cheyne-Stoke } \\
\text { convulsions, coma, décès }\end{array}$ & $250->500$ \\
\hline
\end{tabular}

\section{Les produits dérivés du GHB : $\gamma$-butyrolactone et 1,4-butanediol}

La $\gamma$-butyrolactone (GBL) et le 1,4-butanediol (BD) sont des précurseurs chimiques du GHB. Après le classement sur la liste I des substances contrôlées (schedule I) en 2000, aux États-Unis, la GBL et le BD sont devenus très recherchés. Ce sont deux solvants industriels, que l'on trouve dans le commerce de détail. Après la disparition du GHB légal, ils ont été vendus comme compléments alimentaires $(4,7)$, avec des propriétés alléguées de stimulation de l'hormone de croissance, d'anabolisant, de sédatif léger. Malgré les avertissements de la FDA sur les dangers potentiels de l'ingestion de GBL, seuls trois fabricants sur sept ont cessé leur production et leur vente. Certains l'ont tout simplement remplacé par le $\mathrm{BD}$. On trouve facilement à acheter sur l'Internet les kits et le mode d'emploi pour la fabrication de GHB à partit de GBL ou de BD (24). Les divers noms chimiques, commerciaux et populaires sont donnés dans le tableau II. 
Dans l'organisme la GBL se transforme en GHB par des lactonases. La GBL n'est pas stable dans les flacons et s'y transforme aussi en GHB. La biodisponibilité de la GBL est plus importante que celle du GHB, donnait des concentrations sanguines plus élevées et avec une durée d'action plus longue. C'est le caractère fortement lipophile de la GBL qui lui donne ces propriétés.

Le BD est transformé en 4-hydroxybutanal par l'alcool déshydrogénase, puis en GHB par une aldéhyde déshydrogénase. Il est donc logique que l'ingestion concomitante d'éthanol augmente la toxicité du BD (24). En conclusion, la toxicité aiguë de la GBL, comme celle du $\mathrm{BD}$, est très semblable à celle du $\mathrm{GHB}$, c'est à dire que le risque d'intoxication mortelle existe bel et bien (28).

Tableau II : Noms chimiques et populaires du GHB et de ses dérivés. Certains noms sont communs, les produits pouvant être vendus les uns pour les autres.

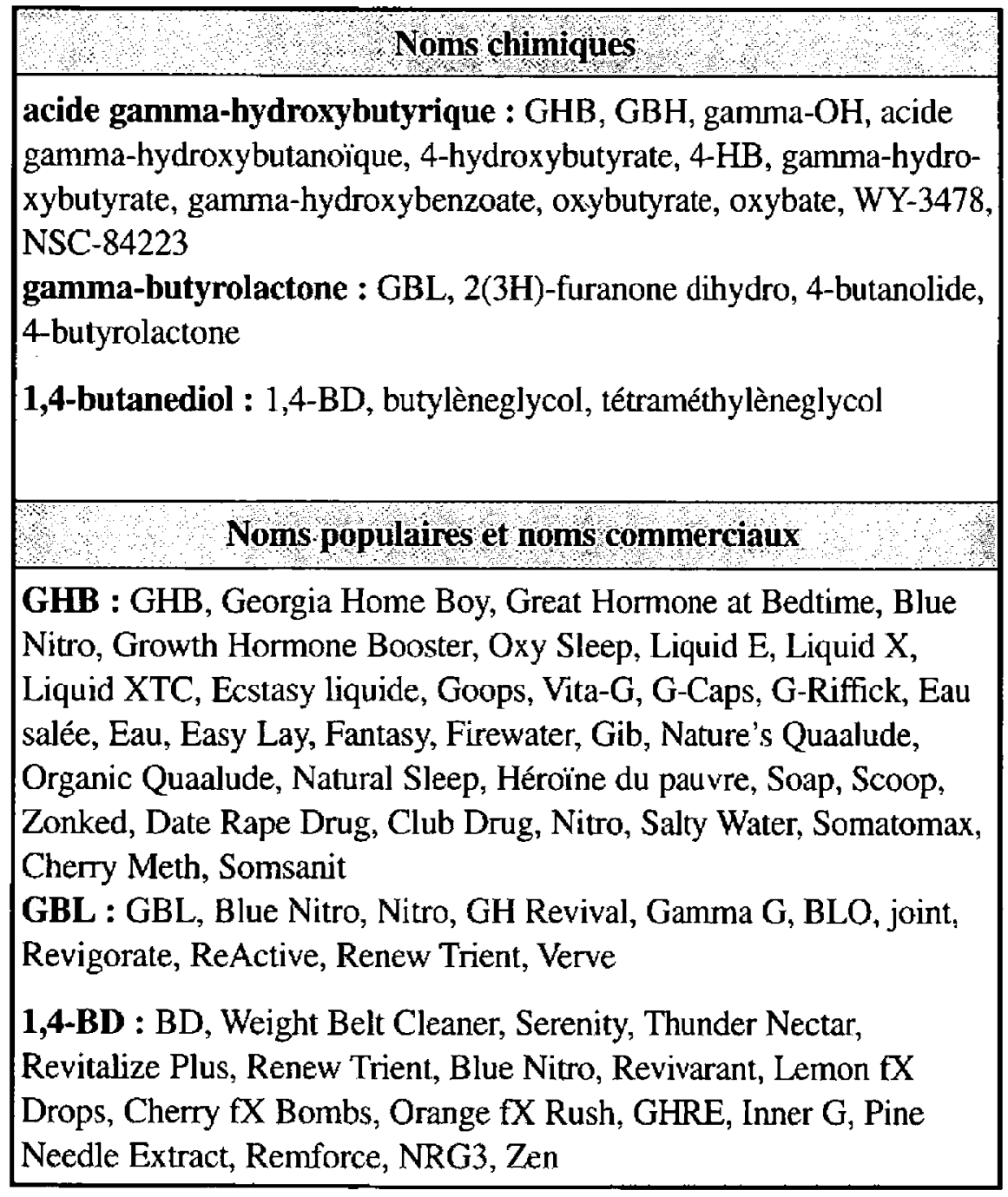

\section{Difficultés posées par le GHB et ses dérivés}

La soumission chimique ou médicamenteuse est encore mal connue des professionnels de santé en France. On la définit par l'administration de substances psychoactives à une victime à son insu, généralement à des fins de viol ou de vol. Le GHB et ses dérivés ont, on l'a vu plus haut, les propriétés nécessaires : absence d'op- position, amnésie antérograde, demi-vie courte (9). Les patients tombant dans un coma soudain et inexpliqué, les femmes pensant avoir été victime d'un viol après avoir ingéré une substance inconnue ayant annihilé leur volonté (réveil dans un lieu inconnu, lésions cutanées, disparition des vêtements) $(7,8)$, doivent pouvoir bénéficier très rapidement d'une recherche et d'un dosage de GHB dans le sang et l'urine. Comme de graves conséquences judiciaires et médico-légales peuvent en découler, il est indispensable de pouvoir poser un diagnostic de certitude.

\section{Difficultés analytiques}

Le dosage lui-même du GHB ne pose a priori pas de difficultés techniques. Cependant il existe de nombreuses méthodes $(2,29)$ et faute de comparaisons inter laboratoires, il est difficile d'affirmer laquelle est la plus juste et la plus précise. On distingue trois types de techniques a) transformation du GHB en GBL puis dosage de la GBL par CPG-SM ; $b$ ) dosage direct par CPG-SM du GHB après dérivation ; $c$ ) dosage du GHB par CLHP.

\section{Transformation du GHB en GBL (30-34)}

La transformation en GBL se fait par addition d'acide sulfurique à chaud $\left(30 \min\right.$ à $\left.90^{\circ} \mathrm{C}\right)$ puis neutralisation par la soude chloroformique. L'extraction est effectuée par un tampon acétate à pH 5,5. La séparation et l'identification se font en CPG-SM (ions d'identification de la GBL : $\mathrm{m} / \mathrm{z} 48,56$ et 86 ). L'acide valproïque et le GHB- $\mathrm{D}_{6}$ sont les étalons interne les plus utilisés, mais plus d'une dizaine ont été testés dont le diéthylèneglycol, l'acide gamma-hydroxyvalérique et l'acide hydroxy-méthylbutyrique. La transformation en GBL et l'échantillonnage peuvent se faire dans le même flacon : on analyse alors l'espace de tête du flacon.

L'inconvénient de cette méthode est la transformation en GBL, qui impose, si l'on veut rechercher spécifiquement cette molécule, de faire deux dosages successifs, avant et après acidification : le volume d'échantillon nécessaire, la durée et le coût deviennent des facteurs limitants. Ces méthodes sont applicables sur l'urine et sur le plasma.

Dosage direct du GHB (revue générale dans 35, 36-39)

De très nombreuse méthodes ont été développées, mais elles restent très proches dans leur principe. La méthode décrite par Verstraete (36) permet de résumer l'essentiel : à $20 \mu \mathrm{L}$ de sérum, de plasma ou d'urine est ajouté l'étalon interne (GHB-D6 6 ou acide valproïque). La déprotéinisation se fait par $45 \mu \mathrm{L}$ d'acétonitrile. Après évaporation douce, on effectue une dérivation par le BSTFA $+1 \%$ TMCS. La séparation est obtenue sur une colonne capillaire de type HP Ultra 1 ou MN Optima $1(12 \mathrm{~m} \times 0,2 \mathrm{~mm}$, film de $0,35 \mu \mathrm{m})$ ou encore 
HP5-MS. L'injecteur en mode splitless est à $250^{\circ} \mathrm{C}$ et la programmation de température du four est la suivante : $50^{\circ} \mathrm{C}$ pendant $0,6 \mathrm{~min}$, puis augmentation de $10^{\circ} \mathrm{C} / \mathrm{min}$ jusqu'à $100^{\circ} \mathrm{C}$ puis de $50^{\circ} \mathrm{C} /$ min jusqu'à $250^{\circ} \mathrm{C}$. Le GHB est identifié par ses ions ( $\mathrm{m} / \mathrm{z} 233,204,159)$.

La comparaison effectuée en 2001 entre plusieurs laboratoires européens (2) a montré que la limite de détection va de 0,5 à $2 \mu \mathrm{g} / \mathrm{mL}$ et la limite de quantification de 2,5 à $5 \mu \mathrm{g} / \mathrm{mL}$. La méthode est linéaire de 2,5 à 200 $\mu \mathrm{g} / \mathrm{mL}$. Elle est reproductible, avec des coefficients de variation de $5,2 \%$ à $5 \mu \mathrm{g} / \mathrm{mL}$ et de $9,4 \%$ à $100 \mu \mathrm{g} / \mathrm{mL}$. Elle est également fidèle : les coefficients de variations sont de $2,7 \%$ à $10 \mu \mathrm{g} / \mathrm{mL}$ et de $4,8 \%$ à $1000 \mu \mathrm{g} / \mathrm{mL}$. Le rendement d'extraction va de 75 à $80 \%$.

Des variantes proposent une laboratoires par le méthanol et une micro extraction en phase solide (40).

\section{Autres méthodes}

De rares auteurs ont proposé de doser le GHB et ses dérivés en CLHP. Une revue des ces méthodes a été effectué récemment par de Vriendt (41). Il est indispensable d'utiliser une colonne d'interaction hydrophile et une micro extraction en phase solide pour obtenir un extrait très propre. La détection se fait par une barrette de photodiodes à $220 \mathrm{~nm}$.

Enfin, des tests colorimétriques rapides peuvent être utilisés pour détecter la présence de GHB ou de GBL dans les urines en cas de suspicion d'intoxication aiguë $(42,43)$. La limite de détection de $0,1 \mathrm{mg} / \mathrm{mL}$, à partir d'un échantillon de $1 \mathrm{~mL}$. Cependant le risque d'interférence avec le GHB endogène urinaire n'est pas totalement exclu. Actuellement, il n'existe pas de test de dépistage immunochimique.

En conclusion, les problèmes analytiques sont faciles à résoudre, la seule difficulté restant dans la séparation chromatographique du GHB, de la GBL et du 1,4-butanediol, chacun de ces produits pouvant être consommé isolément et se transformant l'un dans l'autre.

\section{Difficultés d'interprétation}

\section{Analyse des boissons}

De prime abord, l'interprétation des résultats d'analyse de boissons suspectes est délicate. En effet, Vose (44) a montré que la GBL était un composant naturel de certains vins américains et européens (aux environs de $5 \mu \mathrm{g} / \mathrm{mL}$ ), mais pas du jus de raisin. On sait également qu'en solution aqueuse, le GHB et la GBL s'interconvertissent, dans des rapports variant avec le $\mathrm{pH}(45)$.

\section{Seuils de positivité chez le sujet vivant}

A partir de prélèvements biologiques, l'interprétation des résultats est difficile car le GHB est une substance endogène que l'on retrouve naturellement dans le sang et l'urine. C'est aussi un métabolite du tétrahydrofura- ne, de la GBL et du 1,4-butanediol. Bien qu'il n'existe pas encore de consensus, les concentrations considérées comme physiologiques dans le sang d'individus vivants sont situées entre 1 et $5 \mu \mathrm{g} / \mathrm{mL}$ (46-48). Pan (49) considère même un seuil de $50 \mu \mathrm{g} / \mathrm{mL}$.

L'unanimité ne se fait pas non plus pour les urines et les concentrations considérées comme physiologiques vont de $5 \mu \mathrm{g} / \mathrm{mL}$ (46) à 10 (48) voire $20 \mu \mathrm{g} / \mathrm{mL}$ (34) : elles y sont environ 10 fois plus élevées que dans le sang. Les cheveux permettraient de faire la discrimination en utilisant un seuil de $2 \mathrm{ng} / \mathrm{mg}$, quelle que soit la couleur $(46,48)$. Kintz (48) propose d'analyser des sections de $0,5 \mathrm{~cm}$ à partir du cuir chevelu et de comparer les concentrations observées à la période correspondant aux faits aux autres périodes : l'individu est alors son propre témoin. Des observations récentes montre qu'il serait même possible de vérifier par ce type d'analyse une prise unique un mois avant (Pépin G., communication personnelle).

Il est également possible de doser le GHB dans la sueur et, par comparaison avec les résultats obtenus dans les urines, de mettre ainsi en évidence une ingestion de GHB plus de 12 heures après (urines : $<3 \mu \mathrm{g} / \mathrm{mL}$, sueur : 539 et $649 \mathrm{ng} / \mathrm{patch} / 12 \mathrm{~h}$, avec une concentration physiologique de $242 \mathrm{ng} / \mathrm{patch} / 24 \mathrm{~h}$ ) (48).

\section{Seuils de positivité chez le sujet décédé}

Dans les prélèvements réalisés post mortem le problème semble encore plus complexe, car du GHB peut se former in vitro dans le sang et l'urine $(34,47,50,51)$. Le mode de conservation des échantillons semble alors jouer un rôle très important : le sang devrait être prélevé sur fluorure de sodium, seul ou avec de l'oxalate de potassium, ou mieux sur EDTA, et surtout pas sur citrate $(34,52,53)$. La conservation devra se faire à température constante, $+4^{\circ} \mathrm{C}$ ou $-20^{\circ} \mathrm{C}$, en évitant absolument les congélations et décongélations successives (34). Des concentrations dans le sang de 10 à $197 \mu \mathrm{g} / \mathrm{mL}$ et dans l'urine de 6 à $217 \mu \mathrm{g} / \mathrm{mL}$ ont déjà été retrouvées en l'absence prouvée de toute ingestion de GHB (47). Très récemment, une étude pilote a cependant montré qu'il pouvait y avoir destruction du GHB dans des tubes de sang mal conservés (54). Enfin, une étude expérimentale chez le rat montre que la concentration en GHB dans le foie augmente après le décès (55).

\section{Conclusion}

Une démarche commune pourrait être adoptée, en se fondant sur les résultats obtenus $(19,46,48,49,52$, 56). La prise de GHB sera confirmée par l'ensemble des données suivantes : état du patient décrit par une observation clinique rigoureuse, forte présomption de prise de GHB (produit retrouvé et identifié, témoi- 
gnages de l'entourage direct), concentration sanguine supérieure à $5 \mu \mathrm{g} / \mathrm{mL}$ (sang prélevé sur EDTA), concentration urinaire supérieure à $10 \mu \mathrm{g} / \mathrm{mL}$, concentration dans les cheveux supérieure à $2 \mathrm{ng} / \mathrm{mg}$, recherche dans la sueur positive après $12 \mathrm{~h}$. Cependant, dans les cas de soumission chimique, les doses administrées sont moins fortes que celles utilisées dans les milieux festifs et les délais entre l'administration et les prélèvements encore plus longs : l'interprétation des résultats sera encore plus difficile et les analyses dans les cheveux encore plus importantes.

Il est donc justifié d'établir des protocoles très stricts de prélèvements en concertation avec les services d'urgence (médico-judiciaires ou autres), qui seront par ailleurs formés à ce problème, et enfin de ne confier ces analyses qu'à des laboratoires entraînés et qualifiés. La recherche et le dosage d'autres substances psychotropes ne devront pas être omise (alcool, MDMA et autres amphétamines, benzodiazépines).

Chez un sujet décédé, la conclusion pourrait se faire à partir des seuils suivants, à condition d'avoir pu réaliser les analyses sur tous ces prélèvements : concentration

\section{Références}

1. Laborit H., Jouany J., Gérard J., Fabiani F. Généralités concernant l'étude expérimentale et l'emploi clinique du gammahydroxybutyrate de sodium. Agressologie. 1960 ; 1(4) : 397-406.

2. Deveaux M., Renet S., Renet V., Gaulier J.M., Kintz P., Verstraete A., Gosset D. Utilisation de l'acide gammahydrox ybutyrique (GHB) dans les rave-parties et pour la soumission chimique en France : mythe ou réalité ? Acta Clinica Belgica. 2002 ; 57(Suppl. 1) : 37-40.

3. Nicholson K.L., Balster R.L. GHB : a new and novel drug of abuse. Drug Alcohol Depend. $2001 ; 63$ : 1-22.

4. Smith K.M., Larive L.L., Romanelli F. Club drugs : MDMA, flunitrazepam, ketamine hydrochloride and $\gamma$ hydroxybutyrate. Am. J. Health-Syst.Pharm. 2002 ; 59(1) : 1067-76.

5. Takahara J., Yunoki S., Yakushiji W., Yamauchi W., Yamane Y., Ofuji T. Stimulatory effects of $\gamma$-hydroxybutyric acid on growth hormone and prolactine release in humans. J. Clin. Endrocrinol. Metab. 1977 ; 44 : 1014-7.

6. Dumestre-Toulet V. Se doper par internet ?: un jeu de souris! Ann. Toxicol. Anal. $2000 ; 12(1): 19-25$.

7. Schwartz R.H., Milteer R., LeBeau M.A. Drug-facilitated sexual assault ('date rape'). South. Med. J. 2000 ; 93(6) : 558-61

8. Lapostolle F., Benaissa A., Meuleman C., Jammes D., Buizine A., Baud F. Intoxication aigue involontaire à l'acide $\gamma$-hydroxybutyrique $(\gamma-\mathrm{OH})$. Ann. Fr. Anesth. Réanim. $2001 ; 20 ; 485-7$.

9. Djezzar S, Questel F., Dally S. La soumission médicamenteuse. Courrier Addict. $2001 ; 3(4): 164$.

10. Feigenbaum J.J., Howard S.G. Gamma hydroxybutyrate is not a GABA agonist. Prog. Neurobiol. $1996 ; 50 ; 1-7$. sanguine supérieure à $50 \mu \mathrm{g} / \mathrm{mL}$, concentration urinaire supérieure à $10 \mu \mathrm{g} / \mathrm{mL}$, concentration dans les cheveux supérieure à $2 \mathrm{ng} / \mathrm{mg}$, présence dans le vitré de l'œil.

La dernière étude rassemblant les résultats de 300 recherches de GHB en un an n'a permis de ne retrouver que 3 cas positifs en France et en Belgique (2). On peut donc en déduire que soit il n'est pas présent sur le marché des drogues festives, ce qui ne correspond pas aux dires des jeunes interrogés, soit il est consommé à des doses que l'on peut qualifier de thérapeutiques, sans danger pour les consommateurs, ou enfin que les délais entre l'ingestion et le prélèvement sont trop longs (plus de $4 \mathrm{~h}$ ) et que la concentration de GHB dans le sang et/ou l'urine n'est pas différente de la concentration physiologique. Il serait donc d'un grand intérêt de rassembler les résultats des expertises toxicologiques dans lesquelles du GHB sera retrouvé ou fortement soupçonné d'être présent, afin de mieux cerner les risques associés à la consommation de GHB, en usage récréatif ou abusif, comme cela vient être fait en Australie et aux États-Unis $(3,25)$.

11. Palatini P., Tedeschi L., Frison G., Padrini R., Zordan R., Orlando R., Gallimberti L., Gessa G.L., Ferrara S.D. Dose-dependent absorption and elimination of gammahydroxybutyric acid in healthy volunteers. Eur. J. Pharmacol. $1993 ; 45: 353-6$.

12. Maitre M. The $\gamma$-hydroxybutyrate signalling system in brain : organization and functional implications. Prog. Neurobiol. $1997 ; 51 ; 337-61$.

13. Mamelak M., Scharf M.B., Woods M. Treatment of narcolepsy with gammahydroxybutyrate : a review of clinical and sleep laboratory findings. Sleep. $1986 ; 9: 285-9$.

14. Vickers M.D. Gammahydroxybutyric acid. Int. Anesth. Clin. $1969 ; 7: 75-89$.

15. Ferrara S.D., Zotti S., Tedeschi L., Frison G., Castagna F., Gallimberti L., Gessa G.L., Palatini P. Pharmacokinetics of $\gamma$-hydroxybutyric acid in alcohol dependent patients after single and repeated doses. Br. J. Clin. Pharmacol. $1992 ; 34: 231-5$.

16. Kintz P., Goullé J.P., Cirimele V., Ludes B. Window of detection of GHB in blood and saliva. Clin. Chem. $2001 ; 47: 2033-4$.

17. Dyer J.E. Gamma-hydroxybutyrate : a health food product producing coma and seizure like activity. Am. J. Emerg. Med. ; 1991 ; 9 : 321-4.

18. Stephens G.B., Baselt R.C. Driving under the influence of GHB. J. Anal. Toxicol. $1994 ; 18$ : 357-8.

19. Couper F.J., Logan B.K. Determination of gammahydroxybutyrate (GHB) in biological specimen by GCMS. J. Anal. Toxicol. $2000 ; 24: 1-7$.

20. Hoes M.J., Vree T.B., Guelen P.J. Gamma-hydroxybutyric acid as hypnotic. Clinical and pharmacokinetic evaluation of gamma-hydroxybutyric acid as hypnotic in man. L'Encéphale. $1980 ; 6: 93-9$. 
21. Kavanagh P.V., Kenny P., Fely J. The urinary excretion of gamma-hydroxybutyric acid in man. J. Pharm. Pharmac. $2001 ; 53: 399-402$.

22. Garrison G., Muller P. Clinical features and outcome after unintentional gamma hydroxybutyrate (GHB) overdose. J. Toxicol. Clin. Toxicol. $1998 ; 36: 503-4$.

23. Chin R.I., Sporer K.A., Cullison B., Dyer J.E., Wu T.D. Clinical course of gamma-hydroxybutyrate overdose. Am. Emerg. Med. 1998 ; 31 : 716-22.

24. Shannon M., Quang L.S. Gamma-hydroxybutyrolactone, 1,4-butanediol : a case report and review of the literature. Pediatr. Emerg. Care. 2000 ; 16(6) : 435-40.

25. Degenhardt L., Darke S., Dillon P. GHB use among Australians : characteristics, use patterns and associated harm. Drug Alcohol Depend. 2002 ; 67(1) : 89-94.

26. Bacon E. Drogues : les anciennes et les nouvelles. Act. Méd. Int. Psychiatrie. $2001 ; 18$ (7) : 189-93.

27. Galloway G.P., Frederick S.L., Staggers F.E., Gonzales M., Stalcup S.A., Smith D.E. gamma-hydroxybutyrate : an emerging drug of abuse that causes physical dependency. Addiction. $1997 ; 92$ : 89-96.

28. Zvosec D.L., Smith S.W., McCutcheon J.R., Spillane J., Hall B., Peacock E.A. Adverse effects, including death, associated with the use of 1,4-butanediol. N Engl. J. Med. $2001 ; 344(2): 87-94$.

29. Ghysel M.H. Le GHB (acide gamma-hydroxybutyrique). Revue de la littérature. Toxicorama. $1999 ; 11(1): 1-11$.

30. Doherty J.D., Snead O.C., Roth R.H. A sensitive method for quantitation of $\gamma$-hydroxybutyric acid and $\gamma$-butyrolactone in brain by ECD-GC. Anal. Biochem. 1975;69: 268-77.

31. Vree T.B., van der Kleijn E., Knop H.J. Rapid determination of 4-hydroxybutyric acid (Gamma $\mathrm{OH}$ ) and depakine in human plasma by means of gas-liquid chromatography. J. Chromatrogr. $1976 ; 121: 150-2$.

32. Lettieri J.T., Fung H.L. Evaluation and development of GC procedures for the determination of $\gamma$-hydroxybutyric acid and $\gamma$-butyrolactone. Biochem. Med. 1978;20:70 80.

33. Ferrara S.D., Tedeschi L., Frison G., Castagna F, Gallimberti L., Giorgetti R., Gessa G.L., Palatini P. Therapeutic gamma-hydroxybutyric acid monitoring in plasma and urine by GC-MS. J. Pharm. Biomed. Anal. $1993 ; 11: 483-7$.

34. LeBeau M.A., Montgomery M.A., Miller M.L., Burmeister S.G. Analysis of biofluids for gammahydroxybutyrate (GHB) and gamma-butyrolactone (GBL) by headspace GC-FID and GC-MS. J. Anal. Toxicol. $2000 ; 24: 421-8$.

35.Duer W.C., Byers K.L., Martin J.V. Applicationof a convenient extraction procedure to analyze gammahydroxybutyric acid in fatalities involving gammahydroxybutyric acid, gamma-butyrolactone, and 1,4butanediol. J. Anal. Toxicol, $2001 ; 25: 576-82$.

36. Verstraete A., Van de Velde E., de Paepe P., Rosseel M.T. Determination of gamma-hydroxybutyrate (GHB) in serum or plasma with GC/MS. In : Lech T., ed. Problems of forensic sciences, vol XLII. Cracow : IES. 2000 : 195-201.

37. Elian A.A. A novel method for GHB detection in urine and its application in drug-facilitated sexual assaults. Forensic Sci. Int. $2000 ; 109: 183-7$.
38. Elian A.A. GC-MS determination of gamma-hydroxybutyric acid (GHB) in blood. Forensic Sci. Int. $2001 ; 122$ : 43-7.

39. Kintz P. Difficultés analytiques et d'interprétation dans les expertises toxicologiques liées à la présence de GHB. J. Méd. Lég. $2002 ; 45(6)$ : sous presse.

40. Blair S., Song M., Hall B., Brodbelt J. Determination of gamma-hydroxybutyrate in water and huamn urine by SPME-GC/quadrupole ion trap spectrometry. J Forensic Sci. $2001 ; 46(3)$ : 688-93.

41. de Vriendt C.A., van Sassenbroek D.K., Rosseel M.T., van de Velde E.J., Verstraete A.G., Vander Heyden Y., Belpaire F.M. Development and validation of a HPLC method for the determination of gamma-hydroxybutyric acid in rat plasma. J. Chromatogr. B. $2001 ; 752: 85-80$.

42. Badcock N.R., Zotti R. Rapid screening test for $\gamma$ hydroxybutyric acid (GHB, Fantasy) in urine. Ther. Drug Monit. $1999 ; 21: 376$.

43. Alston II W.C., $\mathrm{Ng} \mathrm{K}$. rapid colorimetric screeninig test for $\gamma$-hydroxybutyrate (ecstasy) in human urine. Forensic Sci. Int.. $2002 ; 133: 1-4$.

44. Vose J., Tighe T., Schwartz M., Buel E. Detection of gamma-butyrolactone (GBL) as a natural component in wine. J. Forensic Sci. 2001 ; 46(5) : 1164-7.

45. Ciolino L.A., Mesmer M.Z., Satzger R.D., Machal A.C., McCauley H.A., Mohrhaus A.S. The chemical interconversion of GHB and GBL: forensic issues and implications. J. Forensic Sci. $2001 ; 46(6)$ : 1315-23.

46. Kalasinsky K.S., Dixon M.M., Schumnk G.A., Kish S.J. Blood, brain and hair GHB concentrations following fatal ingestion. J. Forensic Sci. 2001 ; 46(3) : 728-30.

47. Elliot $S$. The presence of GHB in postmortem biological fluids. J. Anal. Toxicol. $2001 ; 25: 152$.

48. Kintz P., Villain M., Cirimele C., dépendante J.P., Ludes $B$. Usage criminel de substances psychoactives : le problème de la durée de détection. Acta Clinica Belgica. 2002 ; 57(Suppl. 1), 12-5.

49. Pan Y.M., Gill G.N., Tilson C.S., Wall W.H., McCurdy H.H. Improved procedure for the analysis of gammahydroxybutyrate and ethyleneglycol in whole blood. J. Anal. Toxicol. $2001 ; 25: 328-32$.

50. Fieler E.L., Coleman D.E., Baselt R.C. Gamma-hydroxybutyrate concentrations in pre-and postmortem blood and urine. Clin Chem. $1998 ; 44(3): 692$.

51. Ghysel M.H., Dupont V., Salvadore O., Cirimele V., Kintz P. L'intéret du dosage du GHB en toxicologie médico-légale. Toxicorama. $1999 ; 11(3)$ : 195-6.

52. Couper F.J., Logan B.K. GHB and driving impairment. J. Forensic Sci. $2001 ;$ 46(4): 919-23.

53. Kintz P. La soumission chimique. Aspects analytiques et expertises médico-légales. Ann. Toxicol. Anal. 2001 ; $13(2): 120-1$

54. Westerbrinck B. Observations in the quantitative analysis of GHB in a spiked blood sample. Toxi-Logic. 2001 ; 26(1): 10-2.

55. Sakurada K., Kobayashi M., Iwase H., Yoshino M., Mukoyama H., Takatori T., Yoshida K. Production of $\gamma$ hydroxybutyric acid in postmortem liver increases with time after death. Tox. Letter. $2002 ; 129: 207-17$.

56. Karch S.B., Stephens B.G., Nazareno G.V. GHB. Club drug or confusing artifact? Am. J. Forensic Med. Pathol. $2001 ; 22(3): 266-9$. 